\title{
Traitement du petit-lait par osmose inverse
}

\author{
par \\ G. VERNOIS \\ Ingénieur-conseil à Vanves (92)
}

L'utilisation rationnelle du petit-lait provenant de la fabrication du fromage représente souvent un problème n'étant pas encore solutionné d'une façon satisfaisante. Comme l'évacuation du petit-lait renchérit les frais de l'épuration des eaux usées, une utilisation même non profitable peut être indiquée, d'autant plus que la plupart des fromageries déversant le petit-lait dans la rivière la plus proche contribuent ainsi à la pollution de son eau ; mais cette manière de se débarrasser du sous-produit de la fromagerie est maintenant interdite dans de nombreuses régions, car l'évacuation directe dans les rivières provoque des réclamations justifiées à cause de la tendance du petit-lait de fermenter facilement. Par conséquent les fromageries peuvent être astreintes par les offices gouvernementaux de contrôle à ériger des installations pour rendre inoffensives les substances contenues dans le petit-lait [1].

Ce petit-lait contient 4,5 à 5 p. 100 de lactose (sucre de lait), 0,7 p. 100 de sels et seulement 0,8 à 1 p. 100 d'albumine de haute qualité dont la préparation à l'état pur est assez coûteuse, surtout si le petit-lait doit être d'abord dessalé afin d'obtenir une protéine de sérum soluble et non-dénaturée [1].

D'autre part on peut constater [2] que le petit-lait contient environ la moitié des solides présents dans le lait, à savoir la presque totalité du lactose, environ un cinquième des protéines (le lait contient en moyenne 2,9 p. 100 de caséine et 0,5 p. 100 de protéines de sérum, soit une teneur totale en albumine de 3,4 p. 100) et la majeure partie des vitamines et des sels minéraux solubles dans l'eau [2].

\section{Utilisation industrielle du petit-lait [1]}

Les besoins en sucre de lait pouvant être fabriqué économiquement à partir du petit-lait sont plus réduits que l'offre.

Les fabriques de pénicilline ayant consommé autrefois de grandes quantités de lactose pour les bouillons de culture utilisent actuellement le glucose meilleur marché. 
Pour la fabrication de l'acide lactique pur, d'autres hydrates de carbone sont également plus économiques que le sucre de lait ou le petit-lait étant employés en faible quantité seulement comme matière première et alors surtout pour l'obtention de l'acide lactique brut, par exemple pour tanneries.

La production d'alcool par fermentation du petit-lait n'est pas lucrative non plus. Mais une grande partie du petit-lait est utilisée comme aliment de bétail ; lors de la production industrielle des aliments de bétail, le petit-lait fortement concentré (50 à 80 p. 100 de matière sèche) ou la poudre de petit-lait atomisé sont incorporés dans les mélanges de fourrage concentré (par exemple nourriture pour poules).

On peut obtenir également de l'albumine pour matières fourragères en réchauffant le petit-lait à $95^{\circ} \mathrm{C}$, en l'acidifiant et en séparant par centrifugation l'albumine coagulée. Suivant un autre procédé, le petit-lait est fermenté par Lactobac. bulgaricus : la teneur en lactose diminue, tandis que la teneur en azote y augmente jusqu'à 0,8 p. 100. Après neutralisation par $\mathrm{NH}_{3}$, on concentre jusqu'à consistance pâteuse.

La culture de la levure fourragère ou nourricière sur petit-lait a également une certaine importance.

Par contre l'utilisation de produits à base de petit-lait pour l'alimentation humaine n'a pris que des dimensions réduites. A cause du goût salé du petit-lait, la fabrication - facile à concevoir - de limonade n'est que possible après dessalement ou après une forte dilution du petit-lait par sirop de sucre. Le petit-lait concentré à la consistance pâteuse (en y ajoutant éventuellement du lait ou de petites quantités de lait concentré sucré comme semence pour la production de fins cristaux de lactose) est consommé en Norvège comme fromage de petit-lait.

Des quantités peu importantes de petit-lait sont employées à la production d'albumine pour l'alimentation enfantine ainsi qu'à la fabrication de mélanges amino-acides ou sous forme de petit-lait séché comme additif aux sels de fusion (pour fromage).

On a proposé également de précipiter la protéine du petit-lait à l'aide d'acide et de $\mathrm{FeCl}_{3}$ et de l'employer comme " charge » pour plasma sanguin.

\section{Dessalement du petit-lait pour l'alimentation humaine [1]}

Comme nous l'avons vu dans le chapitre précédent, la haute teneur en sels du produit obtenu à partir du petit-lait rend son utilisation très précaire pour l'alimentation humaine. Il est donc évident qu'il serait intéressant de disposer d'un moyen économique pour appauvrir en eau le petit-lait, tout en réduisant sa teneur en sels. 
On a réalisé le dessalement du petit-lait par dialyse (resp. électrodialyse) ou par les procédés d'échange d'ions. La dialyse (resp. l'électrodialyse) permet non seulement de dessaler le petit-lait mais aussi en même temps de réduire la teneur en lactose, c'est-à-dire d'obtenir des produits à teneur accrue en albumine. Mais ces procédés ne sont pas très économiques.

Le procédé d'échange d'ions est utilisé parfois pour le dessalement du petit-lait en vue de la fabrication detla lactose; si ce traitement a lieu après l'extraction de l'albumine, on obtient une lactose d'une teneur moyenne de 97 p. 100 en lactose très pure. On a produit également à partir de petit-lait, par échange d'ions, une préparation de lactose-albumine pratiquement exempte de sel.

Mais ces procédés ne permettent pas le dessalement et la déshydratation du petit-lait en même temps. Par contre, grâce à l'utilisation du phénomène de «l'osmose inverse », on peut obtenir le dessalement et, en même temps, l'appauvrissement en eau du petitlait, donc sa concentration.

\section{Pression osmotique [3] et osmose inverse}

Les substances finement divisées et dissoutes dans un solvant ne peuvent pas en sortir lorsqu'elles proviennent d'une substance non volatile. Par conséquent la vapeur d'eau flottant au-dessus d'une solution saline ne contient rien de la substance dissoute et, par conséquent, cette dernière ne possède pas de pression de vapeur. Par contre les particules de la substance dissoute exercent, à l'intérieur du liquide, qui les retient, une pression pouvant monter éventuellement à beaucoup de bars. Cette pression découverte par Pfeffer (1877) et désignée par " pression osmotique » se manifeste également vers l'extérieur et peut être mesurée à l'aide d'un manomètre, si l'on dispose, entre des quantités adjacentes de la solution et du solvant pur, une membrane du type "semi-perméable " ne laissant passer que le solvant, mais non la substance dissoute. Si cette membrane n'existait pas, la substance dissoute passerait de la solution au solvant pur ; mais elle en est empêchée par la membrane semiperméable, sur laquelle elle exerce alors une pression. Le phénomène d'osmose fait donc que de l'eau pure passe dans la solution saline à travers une membrane semi-perméable jusqu'à ce qu'il s'établisse une différence de niveau représentative de la pression osmotique $\mathrm{p}$ (voir fig. 1) dont la valeur dépend de la concentration atteinte par la solution saline, lorsque s'établit l'équilibre [4].

Pour inverser le mouvement, il suffit d'exercer une pression $\mathrm{P}$ sur la surface de la solution saline qui soit supérieure à la pression osmotique $\mathrm{p}$ (fig. 2). Alors s'établit un écoulement de la solution saline vers l'eau. Peu à peu, le volume d'eau augmente au détriment de celui de la solution saline, la concentration de cette dernière augmentant au fur et à mesure. En d'autres termes, on dessale la 
solution saline pour produire de l'eau pure. Il s'établit ainsi une différence de niveau p' qui représente la différence entre la pression exercée $\mathrm{P}$ et la pression osmotique $\mathrm{p}$.

(1)

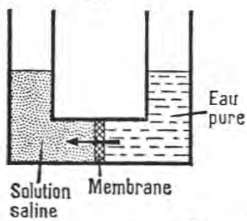

Figure 1

Osmose (document Vichney)
(2)

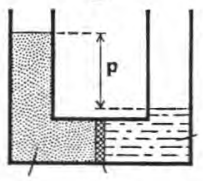

(1)

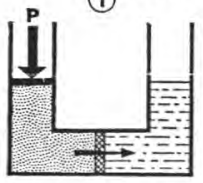

OSMOSE INVERSE

(2)

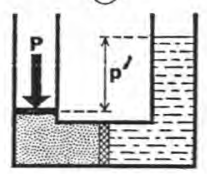

Figure 2

Osmose inverse (document Vichney)

La pression osmotique d'une solution saline peut être mesurée à l'aide d'un osmomètre dont de nombreux systèmes existent ; le plus ancien est l' "endosmomètre » construit par Dutrochet qui s'est basé sur les travaux de l'abbé J. A. Nollet (1700-1770) ; c'est Nollet qui a découvert le phénomène de l' « endosmose ».

Mais on peut aussi calculer approximativement la pression osmotique $\mathrm{p}$ d'une solution aqueuse qui est d'après Van't Hoff [3] :

$$
\mathrm{p}=\frac{\mathrm{i} \cdot \mathrm{R} \cdot \mathrm{T} \cdot \mathrm{c}}{\mathrm{M}} \mathrm{en} \mathrm{kg} / \mathrm{cm}^{2}
$$

où

i degré de dissociation (en général entre 1 et 2 )

$\mathrm{R}$ constante des gaz parfaits $\left(0,0848 \mathrm{kgcm} / \mathrm{mol},{ }^{\circ} \mathrm{C}\right)$

$\mathrm{T}$ température absolue en ${ }^{\circ} \mathrm{K}$

c masse du soluté en $\mathrm{g} / 1$

M poids moléculaire du soluté.

Une solution aqueuse contenant $8 \mathrm{~g}$ de $\mathrm{NaCl}$ par litre exercerait donc, à la température de $20^{\circ} \mathrm{C}$, une pression osmotique :

$$
\mathrm{p}=\frac{1,98 \cdot 0,0848.293 .8}{58,46}=6,73 \mathrm{~kg} / \mathrm{cm}^{2} \text {. }
$$

La discussion de l'équation (1) montre, que la pression osmotique $\mathrm{p}$ monte avec la température $\mathrm{T}$ et la concentration de la solution $\mathrm{c}$.

\section{Débit d'eau épurée par osmose inverse}

Diverses théories ont été proposées pour expliquer le fonctionnement de l'osmose inverse, mais l'expérience aurait confirmé la validité de l'équation suivante [5] :

$$
\mathrm{Q}=\mathrm{K} . \mathrm{S}(\mathrm{P}-\mathrm{p})
$$


Le débit d'eau épurée $\mathrm{Q}$ serait donc directement proportionnel à la différence entre pression appliquée $\mathrm{P}$ et pression osmotique $\mathrm{p}$, au coefficient $\mathrm{K}$ étant fonction, entre autres, du matériau choisi et du procédé de fabrication de la membrane, ainsi qu'à la surface active $\mathrm{S}$ de la membrane.

Par contre W.P. Cooke [2] indique une équation différente de la précédente pour le taux de pénétration des fibres creuses en nylon utilisées dans les filtres «Permasep » de Du Pont de Nemours :

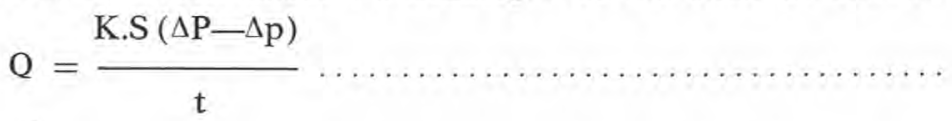

où

$\mathrm{K}$ coefficient de perméabilité à l'eau de la membrane

$S$ surface active de la membrane

$\Delta \mathrm{P}$ différence de pression appliquée sur les deux faces de la membrane

$\Delta \mathrm{p}$ différence de pression osmotique sur les deux faces de la membrane

$\mathrm{t}$ épaisseur de la membrane.

On voit donc que, dans l'équation de Cooke [2], le débit $Q$ est directement proportionnel au coefficient de la perméabilité de la membrane pour une température donnée ainsi qu'à la surface active de cette membrane et à la différence de pression appliquée sur ses deux faces, diminuée de la différence de pression osmotique ; mais il est inversement proportionnel à l'épaisseur de la membrane.

Par conséquent, l'un des objectifs principaux, pour la construction d'un dispositif de séparation par membrane semi-perméable, consisterait à obtenir une surface de membrane aussi grande que possible par unité d'épaisseur, compatible avec la solidité qu'exigent les indispensables différences de pression.

\section{Filtre «Permasep»}

Aux U.S.A. on a développé un système très ingénieux pour la tormation des membranes constituées par des fibres creuses de très faible diamètre et de grande longueur en nylon p.e., dont les extrémités sont scellées dans des plaques « tubulaires » en résine époxy spéciale; une multitude de ces fibres creuses sont disposées dans un récipient sous pression, formé en général par un gros tube. Ce système fut déjà décrit en 1963 par Mahon [6].

Depuis lors, il a fait de grands progrès et la Société Du Pont de Nemours a présenté ce filtre dénommé "Permasep " (marque de fabrique enregistrée également en France) la première fois en mai 1967 à Washington lors de la conférence internationale "L'eau pour la paix». En Europe, ce matériel fut montré la première fois en février 1969 à Rome à l'occasion de l'exposition «Puraqua ». 


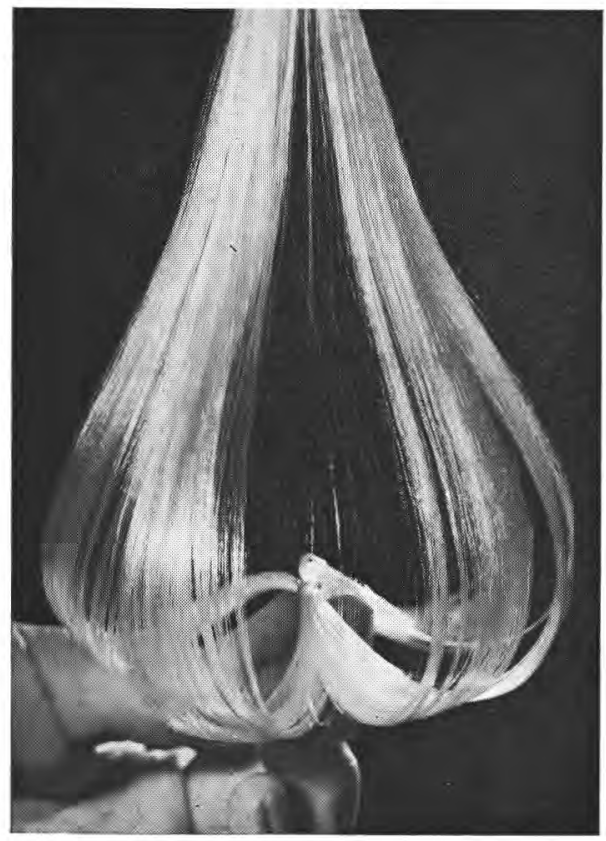

Figure 3

Echeveau de fibres creuses en nylon (document Du Pont)

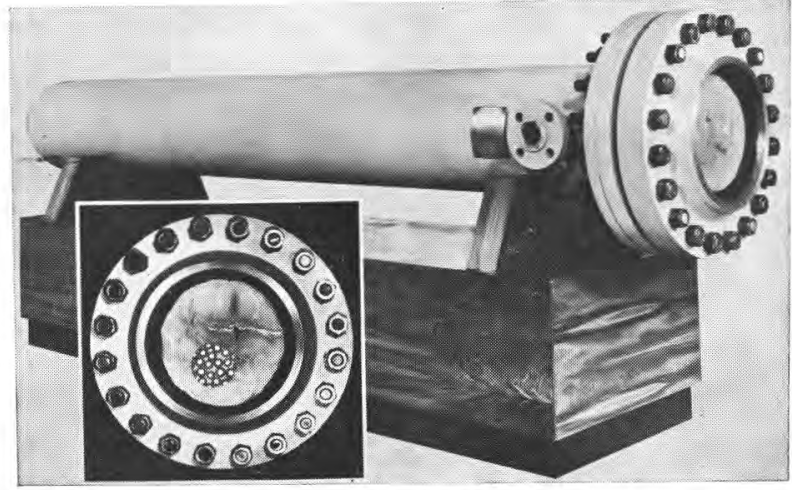

Figure 4

Modèle expérimental du filtre Du Pont « Permasep ", sans la calotte terminale.

Entrée de la solution par la tubulure latérale, sortie du filtrat à l'extrémité des fibres creuses par une tubulure centrale de la calotte terminale.

En gros plan : extrémités ouvertes des fibres creuses. 
Les fibres creuses (fig. 3), plus minces qu'un cheveu humain mais pouvant résister à des pressions de 42 bars, constituent le cœur du filtre Du Pont "Permasep», mis au point pour séparer l'eau ou d'autres solvants des corps dissous qu'ils contiennent. Environ 28000000 de ces fibres creuses robustes et durables se trouvent à l'intérieur du filtre qui se présentent comme des tubes en matière plastique à paroi épaisse et de très petites dimensions (fig. 4).

Le type commercial de ce filtre "Permasep » (fig. 5) est constitué par un tube en acier de $356 \mathrm{~mm}$ de $\varnothing$ et de $3140 \mathrm{~mm}$ de longueur totale ; ses caractéristiques établies à partir de calculs d'optimisation à l'ordinateur et basées sur l'emploi de fibres de nylon spécial sont les suivantes :

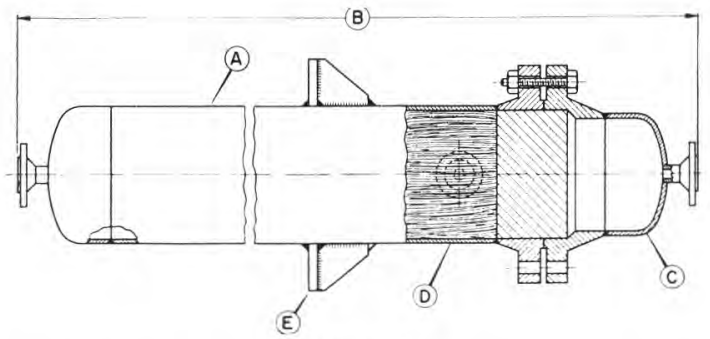

Figure 5

Type commercial du filtre "Permasep» (document Du Pont)

A tube en acier de 14 pouces $(356 \mathrm{~mm})$.

B $3140 \mathrm{~mm}$.

C calotte terminale en acier de 12 pouces $(305 \mathrm{~mm}$ ).

D 2 tubulures d'entrée, décalées de $180^{\circ}$.

E 2 pattes de support.

- diamètre extérieur d'une fibre ..... $0,045 \mathrm{~mm}$

- diamètre intérieur d'une fibre ..... $0,024 \mathrm{~mm}$

— épaisseur de la paroi d'une fibre .... 0,0105 mm

- creux des fibres par rapport à leur section extérieure $\ldots \ldots \ldots \ldots \ldots \ldots$. 28,5 p. 100

- remplissage de l'enveloppe par les fibres $\ldots \ldots \ldots \ldots \ldots \ldots \ldots \ldots \ldots \ldots$. 50 p. 100

- nombre des fibres .............. $28.10^{6}$

— surface filtrante ............... $7850 \mathrm{~m}^{2}$

- capacité du récipient ........... 0,198 $\mathrm{m}^{3}$

- poids net du filtre ............. $680 \mathrm{~kg}$

— débit en eau épurée à $30^{\circ} \mathrm{C} \ldots \ldots .22,7 \mathrm{l} / \mathrm{mn}=32,7 \mathrm{~m}^{3} / 24 \mathrm{~h}$

Le pouvoir sélectif de ce filtre, pour une solution aqueuse à 15 p. 100 de sulfates de sodium et de magnésium (solution mixte standard), sous une pression d'alimentation de 42 bars et pour un taux de transformation de 50 p. 100 de la solution introduite dans le filtre en eau épurée, correspond au passage de 15 p. 100 du soluté à travers la membrane. 
Le passage du soluté est exprimé par l'équation suivante :

$\mathrm{SP}_{\mathrm{E}}=\frac{\mathrm{C}_{\mathrm{P}}}{\mathrm{C}_{\mathrm{F}}} \times 100$

où

$\mathrm{C}_{\mathrm{P}}=$ concentration du soluté dans le filtrat

$\mathrm{C}_{\mathrm{F}}=$ concentration du soluté dans la solution alimentant le filtre.

Il en résulte donc que le dessalement obtenu n'est point total et une petite quantité de sels passe toujours à travers les membranes. Cette quantité varie avec la nature des sels dissous. Ainsi les ions chlore et sodium passent plus facilement à travers les membranes que les ions sulfate et calcium ; l'eau épurée contient donc d'autant moins d'ions que ceux-ci ont une valence plus élevée et un diamètre plus important [5].

Plus le rejet de sels est important, c'est-à-dire plus l'eau produite est épurée, moins la membrane est productrice d'eau par unité de surface.

La récupération, c'est-à-dire le volume d'eau épurée, exprimé en pourcentage du volume de la solution introduite dans le filtre, est présentée par l'équation suivante :

$$
\mathrm{Y}=\frac{\mathrm{V}}{\mathrm{V}}, \times 100
$$

où

V volume d'eau épurée débité par unité de temps $\left(\mathrm{m}^{3} / \mathrm{sec}\right.$.)

$V^{\prime}$ volume de solution alimentant le filtre, débité par la même unité de temps.

D'autre part, d'après l'équation (3), si la solution alimentant le filtre est soumise à une pression augmentée, on doit obtenir un accroissement du débit d'eau épurée, hypothèse que l'expérience a prouvée [2]. Une pression accrue améliore généralement le processus de séparation, puisque contrairement au solvant, le passage du soluté $\left(\mathrm{SP}_{\mathrm{E}}\right)$ n'est pas influencé par la pression. Or, ce dernier étant fonction de la différence de concentration sur les deux faces de la membrane, une récupération plus élevée s'accompagne d'une concentration supérieure du soluté dans l'eau filtrée. Ou en d'autres mots : plus la récupération est forte, plus le passage du soluté dans le filtrat est important.

Pour les filtres «Permasep», les essais effectués ont montré qu'une pression d'alimentation de 42 bars constitue une bonne moyenne et c'est pourquoi il est construit pour une pression nominale de 45,5 bars.

La température a une influence considérable sur le débit des fibres creuses, mais à cause de la résine époxy des plaques " tubulaires ", elle ne devra pas dépasser une limite supérieure de $40^{\circ} \mathrm{C}$ pour une pression de 42 bars. 
A la température de $30^{\circ} \mathrm{C}$, le type commercial du filtre "Permasep » de $356 \mathrm{~mm}$ de $\varnothing$ et de $3140 \mathrm{~mm}$ de longueur a un débit de 15 à $38 \mathrm{~m}^{3} / \mathrm{j}$, exprimé en produit épuré, suivant sélectivité des fibres creuses.

\section{Traitement du petit-lait à l'aide du filtre "Permasep "}

Les premiers résultats des essais effectués jusqu'à présent avec un filtre «Permasep » de $102 \mathrm{~mm}$ de $\varnothing$ et de $2100 \mathrm{~mm}$ de longueur indiquent que les buts envisagés, c'est-à-dire l'appauvrissement en eau du petit-lait et la réduction de sa teneur en sels sont dans le domaine du possible. Actuellement, on évalue les performances du filtre en analysant le petit-lait, le produit de sortie et la solution rejetée, pour pouvoir calculer la demande chimique d'oxygène (D.C.O.) comme moyenne des composés organiques présents, et la conductivité électrique, qui donne la teneur en sels. Les spécialistes de Du Pont de Nemours poursuivent leurs recherches en utilisant des filtres ayant des caractéristiques de sélectivité et de récupération différentes. Mais il est parfaitement possible que l'emploi d'un osmoseur du type "Osmotec », développé en France par un groupe de trois sociétés (Degrémont, S.R.T.I. et S.F.E.C.) et basé aussi sur le principe de l'osmose inverse, permette également une solution du problème de la production d'un concentré de petit-lait à très faible teneur en sels, d'autant plus que les résultats obtenus jusqu'ici en concentration du jus de raisin et de pommes ainsi qu'en dessalement d'eau de mer et des eaux saumâtres ont été remarquables, et ceci avec des solutions essentiellement françaises.

\section{R és u m é}

La haute teneur en sels des produits obtenus ordinairement par le traitement du petit-lait rend leur utilisation impossible pour l'alimentation humaine. Il est donc intéressant de constater qu'on dispose, grâce au procédé de séparation par osmose inverse, d'un moyen économique pour appauvrir en eau le petit-lait, tout en réduisant sa teneur en sels. En effet, l'osmose inverse offre cette possibilité sans élévation notable de température et sans adjonction de produits chimiques ; d'autre part elle ne demande pas la régénération des résines utilisées dans les échangeurs d'ions qui servent parfois au dessalement du petit-lait sans qu'il soit accompagné par l'appauvrissement en eau.

Certes les recherches entreprises jusqu'à maintenant sont encore trop récentes pour permettre un jugement définitif quant aux possibilités offertes par ce nouveau procédé. Toutefois les spécialistes de Du Pont de Nemours sont convaincus que l'osmose inverse offre un moyen souple et économique pour arriver à un résultat intéressant, en réglant le $\mathrm{pH}$, la température et la pression et en utilisant des fibres creuses de différentes structures permettant d'accroître soit le débit, soit la sélectivité. Il est même probable qu'on réussisse, 
dans un proche avenir, à mettre au point des fibres creuses à partir d'autres polymères dont les possibilités de séparation pourraient être infiniment plus étendues que celles des fibres de nylon. Dans ces conditions il est parfaitement possible qu'on puisse transformer très bientôt, à l'aide de l'osmose inverse, le petit-lait en produits se prêtant à l'alimentation humaine.

\section{$\mathrm{S} \mathbf{u} \mathbf{m} \mathbf{m}$ a ry \\ Treatment of whey by reverse osmosis}

The high content of salts in the products obtained usually by the treatment of whey makes impossible its use for the human alimentation. Therefore it is interesting to find out that we dispose owing to the process of separation by reverse osmosis - of an economical way to dehydrate the whey and to reduce its content of salts in the same time. The reverse osmosis gives really this possibility without notable elevation of temperature and without addition of chemical products ; on the other side it does not require the regeneration of the resins employed in the ion-exchangers used sometimes for desalting the whey without being accompanied by loss of water.

Certainly the researches being undertaken till now are too recent in order to permit a definitive opinion relative to the possibilities offered by this new process. However, the specialists of Du Pont de Nemours are convinced that the reverse osmosis offers a supple and economical way in order to arrive to an interesting result by regulation of the $\mathrm{pH}$, the temperature and the pressure and by use of hollow fibres of different structures allowing to increase either the performance or the selectivity. It is even probable to succeed in a near future to realize hollow fibres out of other polymeres whose possibilities of separation might be infinitely more enlarged than those of nylon fibres. In these conditions it is perfectly possible to transform very soon by reverse osmosis the whey into products suitable for human alimentation.

\section{Bibliographie}

[1] Ulumann. - Encyclopédie de la chimie industrielle, $3^{\text {me }}$ édition, vol. 12, Munich-Berlin, 1960.

[2] COOKE (W. P.). - Le rendement des filtres à fibres creuses "Permasep » dans l'épuration et la séparation des eaux usées et des effluents industriels ; Conférence Internationale sur l'épuration et le dessalement des eaux «Puraqua» en février 1969 à Rome.

[3] Schüle (W.). - Thermodynamique industrielle, $4^{\text {me }}$ édition, vol. 1, Berlin, 1921.

[4] Vichney (N.). - Des membranes qui séparent l'eau du sel : le dessalement par osmose inverse. Le Monde du 24 octobre 1968.

[5] Degrémont. - L'osmose inverse (dessalement, purification des eaux, concentration). Paris 1968, notice $\mathrm{n}^{\circ} 1321 \mathrm{~A}$.

[6] Mahon (H.). - Desal. Research Conf. Proc. Publi. 942, 1963.

(Reçu pour publication en juillet 1969). 